PROCEEDINGS OF THE

AMERICAN MATHEMATICAL SOCIETY

Volume 127, Number 6, Pages 1715-1721

S 0002-9939(99)04917-5

Article electronically published on February 11, 1999

\title{
INJECTIVE FACTORIZATION OF HOLOMORPHIC MAPPINGS
}

\author{
MANUEL GONZÁLEZ AND JOAQUÍN M. GUTIÉRREZ
}

(Communicated by Dale Alspach)

\begin{abstract}
We characterize the holomorphic mappings $f$ between complex Banach spaces that may be written in the form $f=g \circ T$, where $g$ is another holomorphic mapping and $T$ is an operator belonging to a closed injective operator ideal. Analogous results are previously obtained for multilinear mappings and polynomials.
\end{abstract}

In recent years, several authors [1], [6], [7] have studied conditions on a holomorphic mapping $f$ between complex Banach spaces so that it may be written in the form $f=g \circ T$, where $g$ is another holomorphic mapping and $T$ is a (linear bounded) operator belonging to certain classes of operators.

In this paper, we look at this problem in the setting of operator ideals, thus finding more general conditions so that these factorizations occur. Our results include all the previous ones, with simpler proofs, and apply to many new cases.

A linear mapping $T$ belongs to the ideal $\mathcal{C} o$ of compact operators if and only if it is weakly (uniformly) continuous on bounded subsets. So, if a mapping $f: E \rightarrow F$ may be written as $f=g \circ T$ with $T \in \mathcal{C} o$, then $f$ is weakly uniformly continuous on bounded subsets of $E$ (the mappings with this property have been studied by numerous authors, for instance [2], [3], [5]). The authors have shown in [7] that the converse also holds: if a holomorphic mapping $f$ between Banach spaces is weakly uniformly continuous on bounded subsets, then it can be factorized in the form $f=g \circ T$, with $T \in \mathcal{C} o$. A similar result was proved in [1] for $T$ in the ideal $\mathcal{W C} o$ of weakly compact operators, and $F=\mathbf{C}$ the complex field.

In [6, Satz 2.1], using interpolation techniques, the factorization $f=g \circ T$ has been characterized in terms of the derivatives of $f$, for $T$ in any closed, injective and surjective operator ideal, and $F=\mathbf{C}$.

In this paper, for any closed injective operator ideal $\mathcal{U}$, we find several conditions on a polynomial $P: E \rightarrow F$ (or a multilinear map) so that it may be written as $P=Q \circ T$, with $T$ in $\mathcal{U}$. As a consequence, we prove that a holomorphic map $f: E \rightarrow F$ admits such a factorization if and only if $f$ is uniformly continuous on bounded sets with respect to a suitable topology $\tau_{\mathcal{U}}$. In the case $\mathcal{U}=\mathcal{C}$ o, the topology $\tau_{\mathcal{U}}$ is the finest l.c. topology that coincides with the weak topology on bounded sets. Consequently, using a simpler proof, we recover the result in [7].

Received by the editors September 10, 1997.

1991 Mathematics Subject Classification. Primary 46G20; Secondary 47D50.

Key words and phrases. Factorization, holomorphic mapping between Banach spaces, multilinear mapping, polynomial, operator ideal.

The first author was supported in part by DGICYT Grant PB 97-0349 (Spain).

The second author was supported in part by DGICYT Grant PB 96-0607 (Spain).

(c)1999 American Mathematical Society 
Many usual operators ideals are closed and injective: for instance (see [14]), the (weakly) compact operators, the (weakly) completely continuous operators, the Rosenthal operators, the unconditionally converging operators, the (weakly) Banach-Saks operators [11, §3], the strictly singular operators, the operators with separable range, the decomposing (Asplund) operators, the Radon-Nikodym operators, and the absolutely continuous operators $[12, \S 3]$. Our results apply to all of them.

The conditions that we find so that the factorization occurs are quite natural and have been used by many authors in various contexts (see, e.g., $[1,2,6,8]$ ).

Throughout, $E, F$ and $G$ will denote Banach spaces, and $B_{E}$ will denote the closed unit ball of $E$. When we deal with holomorphic mappings, these spaces will be complex. Otherwise, they can be either real or complex.

We denote by $\mathcal{L}(E, F)$ the space of all operators from $E$ into $F$, endowed with the usual operator norm. If $E_{1}, \ldots, E_{k}$ are also Banach spaces, the notation $\mathcal{L}^{k}\left(E_{1}, \ldots, E_{k} ; F\right)$ stands for the space of all (continuous) $k$-linear mappings from $E_{1} \times \cdots \times E_{k}$ into $F$.

We shall use the notational convention.$[i]$. to mean that the $i$ th coordinate is not involved. For instance,

$$
\mathcal{L}^{k-1}\left(E_{1},{ }^{[i]} ., E_{k} ; F\right):=\mathcal{L}^{k-1}\left(E_{1}, \ldots, E_{i-1}, E_{i+1}, \ldots, E_{k}\right) .
$$

To each $A \in \mathcal{L}^{k}\left(E_{1}, \ldots, E_{k} ; F\right)$ and $i \in\{1, \ldots, k\}$ we associate an operator

$$
A_{i}: E_{i} \longrightarrow \mathcal{L}^{k-1}\left(E_{1},{ }^{[i]} ., E_{k} ; F\right)
$$

given by

$$
A_{i}\left(x_{i}\right)\left(x_{1}, .{ }^{[i]} ., x_{k}\right):=A\left(x_{1}, \ldots, x_{k}\right) \quad\left(x_{j} \in E_{j} ; 1 \leq j \leq k\right) .
$$

The space of all (continuous) $k$-homogeneous polynomials from $E$ into $F$ is denoted by $\mathcal{P}\left({ }^{k} E, F\right)$. To each $P \in \mathcal{P}\left({ }^{k} E, F\right)$ we associate an operator

$$
\bar{P}: E \longrightarrow \mathcal{P}\left({ }^{k-1} E, F\right)
$$

given by $\bar{P}(x)(y):=\hat{P}(x, y, \stackrel{(k-1)}{\cdots}, y)$ where $\hat{P}$ is the unique symmetric, $k$-linear mapping associated to $P$.

For complex spaces $E$ and $F, \mathcal{H}(E, F)$ will denote the space of all holomorphic mappings from $E$ into $F$, and $\mathcal{H}_{b}(E, F)$ will denote the subspace of all $f \in \mathcal{H}(E, F)$ which are bounded on bounded sets. In the complex case too, we say that a subset $A \subset E$ is circled if for every $x \in A$ and complex $\lambda$ with $|\lambda|=1$, we have $\lambda x \in A$.

For a general introduction to polynomials and holomorphic mappings, the reader is referred to $[4,13]$. The definition and general properties of operator ideals may be seen in [14].

An operator ideal $\mathcal{U}$ is said to be injective $[14,4.6 .9]$ if given an operator $T \in$ $\mathcal{L}(E, F)$ and an injective isomorphism $i: F \rightarrow G$, we have that $T \in \mathcal{U}$ whenever $i T \in \mathcal{U}$. We say that $\mathcal{U}$ is closed $[14,4.2 .4]$ if, for all $E$ and $F$, the space $\mathcal{U}(E, F)$ is closed in $\mathcal{L}(E, F)$.

The following basic result will be used:

Lemma 1 ([9, Theorem 20.7.3]). An operator ideal $\mathcal{U}$ is closed and injective if and only if for an operator $T \in \mathcal{L}(E, F)$ to belong to $\mathcal{U}$ it is both necessary and sufficient that for each $\epsilon>0$ there exist a Banach space $G_{\epsilon}$ and an operator $S_{\epsilon} \in \mathcal{U}\left(E, G_{\epsilon}\right)$ so that

$$
\|T x\| \leq\left\|S_{\epsilon}(x)\right\|+\epsilon\|x\| \quad(x \in E) .
$$


Considering now multilinear mappings, we first wish to relate some of their topological properties to those of the associated operators. To this end, we need the following result, whose proof, which is standard, is given for completeness.

Proposition 2. Let $\tau_{i}$ be a vector topology on a Banach space $E_{i}(1 \leq i \leq k)$. Given a $k$-linear mapping $A \in \mathcal{L}^{k}\left(E_{1}, \ldots, E_{k} ; F\right)$ with associated operators $A_{i}$ : $E_{i} \rightarrow \mathcal{L}^{k-1}\left(E_{1},{ }^{[i]} \cdot, E_{k} ; F\right)$, we have that $A$ is uniformly $\tau_{1} \times \cdots \times \tau_{k}$-continuous on bounded sets if and only if the operators $A_{i}$ are (uniformly) $\tau_{i}$-continuous on bounded sets.

Proof. Suppose $A$ is uniformly $\tau_{1} \times \cdots \times \tau_{k}$-continuous on bounded sets. Given $\epsilon>0$, we can find $\tau_{i}$ zero neighbourhoods $U_{i} \subset E_{i}$ so that $\| A\left(x_{1}, \ldots, x_{k}\right)-$ $A\left(y_{1}, \ldots, y_{k}\right) \|<\epsilon$ whenever $x_{i}, y_{i} \in B_{E_{i}}$ satisfy $x_{i}-y_{i} \in U_{i}(1 \leq i \leq k)$. Take $j \in\{1, \ldots, k\}, z_{i} \in B_{E_{i}}$ for $i=1,\left[{ }^{[j]} ., k\right.$, and $x_{j}, y_{j}$ as above. Then

$\left\|\left(A_{j} x_{j}-A_{j} y_{j}\right)\left(z_{1},{ }^{[j]} ., z_{k}\right)\right\|=\left\|A\left(z_{1}, \ldots, x_{j}, \ldots, z_{k}\right)-A\left(z_{1}, \ldots, y_{j}, \ldots, z_{k}\right)\right\|<\epsilon$.

Hence, $A_{j}$ is uniformly $\tau_{j}$-continuous on bounded sets.

Conversely, let $A_{i}$ be $\tau_{i}$-continuous on bounded sets, for $1 \leq i \leq k$. Given $\epsilon>0$, there is a $\tau_{i}$ zero neighbourhood $U_{i} \subset E_{i}$ so that $\left\|A_{i} x-A_{i} y\right\|<\epsilon$ whenever $x, y \in B_{E_{i}}$ satisfy $x-y \in U_{i}$. Given $x_{i}, y_{i} \in B_{E_{i}}$ with $x_{i}-y_{i} \in U_{i}(1 \leq i \leq k)$, we have

$$
\begin{aligned}
& \left\|A\left(x_{1}, \ldots, x_{k}\right)-A\left(y_{1}, \ldots, y_{k}\right)\right\| \\
& \quad \leq\left\|A_{1}\left(x_{1}-y_{1}\right)\left(x_{2}, \ldots, x_{k}\right)\right\|+\left\|A_{2}\left(x_{2}-y_{2}\right)\left(y_{1}, x_{3}, \ldots, x_{k}\right)\right\|+\cdots \\
& \quad+\left\|A_{k}\left(x_{k}-y_{k}\right)\left(y_{1}, \ldots, y_{k-1}\right)\right\| \\
& \quad \leq k \epsilon
\end{aligned}
$$

and so, $A$ is uniformly $\tau_{1} \times \cdots \times \tau_{k}$-continuous on bounded sets.

Let $\mathcal{U}$ be an injective operator ideal. On every Banach space $E$ we consider the topology $\tau_{\mathcal{U}}$ generated by the seminorms

$$
p_{T}(x):=\|T x\|, \quad \text { for } T \in \mathcal{U}(E, F), F \text { any Banach space. }
$$

We then have (see $[10, \S 3])$ :

$$
\mathcal{U}(E, F)=\mathcal{L}\left(\left(E, \tau_{\mathcal{U}}\right), F\right) .
$$

Proposition 3. Given a closed injective ideal $\mathcal{U}$ and $S \in \mathcal{L}(E, F)$, we have that $S \in \mathcal{U}(E, F)$ if and only if $S$ is $\tau_{\mathcal{U}}$-continuous on bounded subsets.

Proof. Note that, since $\mathcal{U}$ is closed, $\tau_{\mathcal{U}}$ is the finest locally convex topology that agrees with $\tau_{\mathcal{U}}$ on bounded subsets (see [10, Proposition 4.2]). Then apply (1).

One of our key results is the next one.

Theorem 4. Given $A \in \mathcal{L}^{k}\left(E_{1}, \ldots, E_{k} ; F\right)$, let $\mathcal{U}$ be a closed injective operator ideal. The following assertions are equivalent: $\mathcal{U}$;

(a) for every $1 \leq i \leq k$, the operator $A_{i}: E_{i} \rightarrow \mathcal{L}^{k-1}\left(E_{1}, .{ }^{[i]} ., E_{k} ; F\right)$ belongs to

(b) there are Banach spaces $Y_{i}$ and operators $T_{i} \in \mathcal{U}\left(E_{i}, Y_{i}\right)(1 \leq i \leq k)$ so that $\left\|A\left(x_{1}, \ldots, x_{k}\right)\right\| \leq\left\|T_{1} x_{1}\right\| \cdot \ldots \cdot\left\|T_{k} x_{k}\right\|$;

(c) there are Banach spaces $Y_{i}$, operators $T_{i} \in \mathcal{U}\left(E_{i}, Y_{i}\right)(1 \leq i \leq k)$ and a mapping $D \in \mathcal{L}^{k}\left(Y_{1}, \ldots, Y_{k} ; F\right)$ so that $A=D \circ\left(T_{1}, \ldots, T_{k}\right)$;

(d) $A$ is uniformly $\tau_{\mathcal{U}}$-continuous on bounded subsets. 
Proof. (a) $\Rightarrow$ (b). Consider the seminorms

$$
q_{i}(x):=\inf _{n \in \mathbf{N}}\left\{n^{k-1}\left\|A_{i} x\right\|+n^{-1}\|x\|\right\} \quad\left(x \in E_{i}\right)
$$

for $1 \leq i \leq k$. Letting $Y_{i}$ be the completion of $\left(E_{i} / \operatorname{ker}\left(q_{i}\right), q_{i}\right)$, define $T_{i}: E_{i} \rightarrow Y_{i}$ by $T_{i} x:=x+\operatorname{ker}\left(q_{i}\right)$, for $x \in E_{i}$. For every $n \in \mathbf{N}$ and $1 \leq i \leq k$, we have

$$
\left\|T_{i} x\right\|=q_{i}(x) \leq n^{k-1}\left\|A_{i} x\right\|+n^{-1}\|x\| \quad\left(x \in E_{i}\right) .
$$

By Lemma 1 we have that $T_{i} \in \mathcal{U}$ for $1 \leq i \leq k$.

It is enough to show that given $x_{i} \in E_{i}$ with $\left\|T_{i} x_{i}\right\|<1$, for $1 \leq i \leq k$, we have $\left\|A\left(x_{1}, \ldots, x_{k}\right)\right\|<1$.

Given $i \in\{1, \ldots, k\}$, there is $n_{i} \in \mathbf{N}$ so that

$$
n_{i}^{k-1}\left\|A_{i} x_{i}\right\|+n_{i}^{-1}\left\|x_{i}\right\|<1 .
$$

Assume $n_{j}=\max \left\{n_{1}, \ldots, n_{k}\right\}$. We have $\left\|x_{i}\right\|<n_{j}$ for all $1 \leq i \leq k$. Moreover, $\left\|A_{j} x_{j}\right\|<n_{j}^{1-k}$. Hence,

$$
\left\|A\left(x_{1}, \ldots, x_{k}\right)\right\|=\left\|A_{j} x_{j}\left(x_{1},[j], x_{k}\right)\right\| \leq\left\|A_{j} x_{j}\right\| \cdot\left\|x_{1}\right\| \cdot \cdot[j] \cdot\left\|x_{k}\right\|<1 .
$$

(b) $\Rightarrow$ (c). Since $\mathcal{U}$ is injective, we can assume that $T_{i}\left(E_{i}\right)$ is dense in $Y_{i}$, for $1 \leq i \leq k$. Define

$$
D\left(T_{1} x_{1}, \ldots, T_{k} x_{k}\right):=A\left(x_{1}, \ldots, x_{k}\right) \quad\left(x_{i} \in E_{i}, 1 \leq i \leq k\right) .
$$

If $T_{i} x_{i}=T_{i} x_{i}^{\prime}$ for all $1 \leq i \leq k$, we then have

$$
\begin{aligned}
A\left(x_{1}, \ldots, x_{k}\right)-A\left(x_{1}^{\prime}, \ldots, x_{k}^{\prime}\right) & \\
= & A\left(x_{1}-x_{1}^{\prime}, x_{2}, \ldots, x_{k}\right)+A\left(x_{1}^{\prime}, x_{2}-x_{2}^{\prime}, \ldots, x_{k}\right) \\
& +\cdots+A\left(x_{1}^{\prime}, \ldots, x_{k-1}^{\prime}, x_{k}-x_{k}^{\prime}\right) \\
= & 0,
\end{aligned}
$$

since $T_{i}\left(x_{i}-x_{i}^{\prime}\right)=0$ for $1 \leq i \leq k$. So, $D$ is well defined and continuous, with $\|D\| \leq 1$. Hence we can extend it to $Y_{1} \times \cdots \times Y_{k}$ and, denoting the extension by $D$ as well, we have $A=D \circ\left(T_{1}, \ldots, T_{k}\right)$.

(c) $\Rightarrow$ (a). Assume $A=D \circ\left(T_{1}, \ldots, T_{k}\right)$ with $T_{i} \in \mathcal{U}\left(E_{i}, Y_{i}\right)$, for $1 \leq i \leq k$. For each $i$, define

$$
B_{i}: Y_{i} \longrightarrow \mathcal{L}^{k-1}\left(E_{1}, .\left[{ }^{[i]} ., E_{k} ; F\right)\right.
$$

by

$$
\left(B_{i} y\right)\left(u_{1}, .\left[{ }^{i}\right] ., u_{k}\right)=D\left(T_{1} u_{1}, \ldots, \stackrel{(i)}{y}, \ldots, T_{k} u_{k}\right) \quad\left(y \in Y_{i} ; u_{1} \in E_{1}, .\left[{ }^{i}\right] ., u_{k} \in E_{k}\right) .
$$

Clearly, $B_{i}$ is continuous, with

$$
\left\|B_{i}\right\| \leq\left\|T_{1}\right\| \cdot[i] \cdot \cdot\left\|T_{k}\right\| \cdot\|D\|,
$$

and $A_{i}=B_{i} \circ T_{i}$. Hence $A_{i} \in \mathcal{U}$.

(a) $\Leftrightarrow$ (d) by Propositions 2 and 3 .

If $\mathcal{U}$ is the ideal of compact operators, then the assertion (d) of the Theorem states that $A$ is weakly uniformly continuous on bounded sets. The result in the compact case was proved in [7].

It is an open problem to characterize the ideals $\mathcal{U}$ such that every $k$-linear mapping which is $\tau_{\mathcal{U}}$-continuous on bounded sets is also uniformly $\tau_{\mathcal{U}}$-continuous on bounded sets. It is a well-known result, proved in [2], that for $\mathcal{U}=\mathcal{C} o$ the assertion 
is true. It is obviously true if $\mathcal{U}$ is the ideal of all bounded operators. We proved in [7] that it fails for $\mathcal{U}=\mathcal{W C} o$, and also for $\mathcal{U}$ the completely continuous operators.

Corollary 5. Given $P \in \mathcal{P}\left({ }^{k} E, F\right)$, let $\mathcal{U}$ be a closed injective operator ideal. The following assertions are equivalent:

(a) the operator $\bar{P}: E \rightarrow \mathcal{P}\left({ }^{k-1} E, F\right)$ belongs to $\mathcal{U}$;

(b) there are a Banach space $Y$ and an operator $T \in \mathcal{U}(E, Y)$ so that $\|P(x)\| \leq$ $\|T(x)\|^{k}$ for all $x \in E$;

(c) there are a Banach space $Y$, an operator $T \in \mathcal{U}(E, Y)$ and a polynomial $Q \in \mathcal{P}\left({ }^{k} Y, F\right)$ so that $P=Q \circ T$;

(d) $P$ is uniformly $\tau_{\mathcal{U}}$-continuous on bounded sets.

The argument in the proof of [4, Lemma 1.16] can be used to show that (b) $\Rightarrow$ (c). The other parts are obtained by adapting the proof of Theorem 4 .

Next, we shall extend the factorization theorem to holomorphic mappings. The following result will be useful:

Lemma 6 ([6, Lemma 1]). Suppose $A=D \circ\left(T_{1}, \ldots, T_{k}\right)$, where $A \neq 0, D \in$ $\mathcal{L}^{k}\left(Y_{1}, \ldots, Y_{k} ; F\right)$, and $T_{i} \in \mathcal{L}\left(E_{i}, Y_{i}\right)$ for $1 \leq i \leq k$. Then the spaces $Y_{i}$ may be renormed so that $\|A\|=\|D\| \cdot\left\|T_{1}\right\| \cdot \ldots \cdot\left\|T_{k}\right\|$.

Proposition 7. Let $\mathcal{U}$ be a closed injective operator ideal. If $f \in \mathcal{H}(E, F)$ is uniformly $\tau_{\mathcal{U}}$-continuous on bounded sets, $a \in E$ and $k \in \mathbf{N}$, then $d^{k} f(a)$ is uniformly $\tau_{\mathcal{U}}$-continuous on bounded sets.

Proof. From the Cauchy integral formula [13, Corollary 7.3], we obtain

$$
\begin{aligned}
\left\|\frac{1}{k !} d^{k} f(a)(x)-\frac{1}{k !} d^{k} f(a)(y)\right\| & =\left\|\frac{1}{2 \pi i} \int_{|\lambda|=1} \frac{f(a+\lambda x)-f(a+\lambda y)}{\lambda^{k+1}} d \lambda\right\| \\
& \leq \sup _{|\lambda|=1}\|f(a+\lambda x)-f(a+\lambda y)\| .
\end{aligned}
$$

Lef $B$ be a circled, bounded subset of $E$. Given $\epsilon>0$, there is a circled $\tau_{\mathcal{U}}$ zero neighbourhood $U$ in $E$ so that $\left\|f\left(x^{\prime}\right)-f\left(y^{\prime}\right)\right\|<\epsilon$ whenever $x^{\prime}, y^{\prime} \in a+B$ satisfy $x^{\prime}-y^{\prime} \in U$. Therefore, given $x, y \in B$ with $x-y \in U$, we easily get from the above inequality:

$$
\left\|\frac{1}{k !} d^{k} f(a)(x)-\frac{1}{k !} d^{k} f(a)(y)\right\|<\epsilon .
$$

If $\left(Y_{k}\right)$ is a sequence of Banach spaces, we denote by $c_{0}\left(Y_{k}\right)$ the Banach space of all sequences $\left(y_{k}\right)$ so that $y_{k} \in Y_{k}$ and $\left\|y_{k}\right\| \rightarrow 0$, endowed with the supremum norm. In the proof of the following Theorem we shall use the well-known fact that if $B \subset E$ is bounded and $f: B \rightarrow F$ is a uniformly continuous mapping, then $f(B)$ is bounded in $F$.

Theorem 8. Given $f \in \mathcal{H}(E, F)$, let $\mathcal{U}$ be a closed injective operator ideal. Then $f$ is uniformly $\tau_{\mathcal{U}}$-continuous on bounded subsets if and only if there are a Banach space $G$, an operator $T \in \mathcal{U}(E, G)$ and a mapping $g \in \mathcal{H}_{b}(G, F)$ so that $f=g \circ T$.

Proof. Suppose $f=g \circ T$, with $g$ and $T$ as in the statement. Let $A \subset E$ be bounded. Then $T$ is uniformly continuous from $\left(A, \tau_{\mathcal{U}}\right)$ into $(T A,\|\cdot\|)$. Since $g$ is bounded on bounded sets, it is norm-to-norm uniformly continuous on bounded sets. Hence, $f$ is uniformly continuous from $\left(A, \tau_{\mathcal{U}}\right)$ into $(F,\|\cdot\|)$. 
Conversely, suppose $f$ is uniformly $\tau_{\mathcal{U}}$-continuous on bounded sets. We write the Taylor series expansion of $f$ at the origin as

$$
f(x)=\sum_{k=0}^{\infty} P_{k}(x) \quad(x \in E) .
$$

By Proposition $7, P_{k}$ is uniformly $\tau_{\mathcal{U}}$-continuous on bounded sets, for each $k$. By Theorem 4, there are a Banach space $Y_{k}$, an operator $T_{k} \in \mathcal{U}\left(E, Y_{k}\right)$ and a polynomial $Q_{k} \in \mathcal{P}\left({ }^{k} Y_{k}, F\right)$ such that $P_{k}=Q_{k} \circ T_{k}$. We can get $\left\|\hat{P}_{k}\right\|=\left\|\hat{Q}_{k}\right\| \cdot\left\|T_{k}\right\|^{k}$ (Lemma 6). Since $\tau_{\mathcal{U}}$ is coarser than the norm topology, it follows that $f \in$ $\mathcal{H}_{b}(E, F)$, and so $\lim \left\|P_{k}\right\|^{1 / k}=0$. From the inequalities

$$
\left\|P_{k}\right\| \leq\left\|\hat{P}_{k}\right\| \leq \frac{k^{k}}{k !}\left\|P_{k}\right\|
$$

(see [13, Theorem 2.2]), and using the Stirling formula, we get $\lim \left\|\hat{P}_{k}\right\|^{1 / k}=0$. Therefore, we can assume that $\left\|\hat{Q}_{k}\right\|^{1 / k} \rightarrow 0$ and $\left\|T_{k}\right\| \rightarrow 0$. Define

$$
T: E \longrightarrow Y:=c_{0}\left(Y_{k}\right)
$$

by $T x:=\left(T_{k} x\right)_{k}$. Clearly, $T \in \mathcal{U}$. Denoting

$$
\pi_{k}:\left(y_{i}\right) \in Y \longmapsto y_{k} \in Y_{k},
$$

we define $g: Y \rightarrow F$ by $g(y):=\sum_{k=1}^{\infty} Q_{k} \circ \pi_{k}(y)$. Since $\lim \left\|Q_{k} \circ \pi_{k}\right\|^{1 / k}=$ $\lim \left\|Q_{k}\right\|^{1 / k}=0$, we get that $g$ is a holomorphic mapping of bounded type that satisfies the requirement.

To finish up, we give a polynomial $P \in \mathcal{P}\left({ }^{3} \ell_{\infty}\right)$ that cannot be written in the form $P=Q \circ S$, with $S \in \mathcal{W C}$. Note that many classes of operators on $\ell_{\infty}$ coincide, e.g., the weakly compact operators, the completely continuous operators, the unconditionally converging operators, etc.

Consider a surjective operator $q: \ell_{\infty} \rightarrow \ell_{2}$ such that $q\left(B_{\ell_{\infty}}\right) \supseteq B_{\ell_{2}}$. Letting $q(x)_{i}$ be the $i$ th coordinate of $q(x) \in \ell_{2}$, we define

$$
P(x):=\sum_{i=1}^{\infty} x_{i} q(x)_{i}^{2}, \quad \text { for } x=\left(x_{i}\right) \in \ell_{\infty} .
$$

The associated operator $\bar{P}: \ell_{\infty} \rightarrow \mathcal{P}\left({ }^{2} \ell_{\infty}\right)$ is given by

$$
\bar{P}(x)(y):=\frac{1}{3} \sum_{i=1}^{\infty} x_{i} q(y)_{i}^{2}+\frac{2}{3} \sum_{i=1}^{\infty} y_{i} q(x)_{i} q(y)_{i} .
$$

We only have to show that $\bar{P} \notin \mathcal{W C}$ o (see Corollary 5) or, equivalently, that $\bar{P}$ is not completely continuous. Denoting by $e_{n}$ the sequence $(0, \ldots, 0,1,0, \ldots)$ with 1 in the $n$th position, we select a sequence $\left(x^{n}\right) \subset B_{\ell_{\infty}}$ so that $q\left(x^{n}\right)=e_{n}$. Then,

$$
3 \bar{P}\left(e_{n}\right)\left(x^{n}\right)=1+2 x_{n}^{n} q\left(e_{n}\right)_{n} .
$$

Since $q\left(e_{n}\right) \rightarrow 0$, we have $3 \bar{P}\left(e_{n}\right)\left(x^{n}\right) \rightarrow 1$ and, therefore, $\left\|\bar{P}\left(e_{n}\right)\right\|$ does not converge to 0 . 


\section{REFERENCES}

[1] R. M. Aron and P. Galindo, Weakly compact multilinear mappings, Proc. Edinburgh Math. Soc. 40 (1997), 181-192. CMP 97:09

[2] R. M. Aron, C. Hervés and M. Valdivia, Weakly continuous mappings on Banach spaces, J. Funct. Anal. 52 (1983), 189-204. MR 84g:46066

[3] R. M. Aron and J. B. Prolla, Polynomial approximation of differentiable functions on Banach spaces, J. Reine Angew. Math. 313 (1980), 195-216. MR 81c:41078

[4] S. Dineen, Complex Analysis in Locally Convex Spaces, Math. Studies 57, North-Holland, Amsterdam 1981. MR 84b:46050

[5] S. Dineen, Entire functions on $c_{0}$, J. Funct. Anal. 52 (1983), 205-218. MR 85a:46024

[6] S. Geiss, Ein Faktorisierungssatz für multilineare Funktionale, Math. Nachr. 134 (1987), 149-159. MR 89b:47067

[7] M. González and J. M. Gutiérrez, Factorization of weakly continuous holomorphic mappings, Studia Math. 118 (1996), 117-133. MR 97b:46061

[8] M. González, J. M. Gutiérrez and J. G. Llavona, Polynomial continuity on $\ell_{1}$, Proc. Amer. Math. Soc. 125 (1997), 1349-1353. MR 97g:46024

[9] H. Jarchow, Locally Convex Spaces, B. G. Teubner, Stuttgart 1981. MR 83h:46008

[10] H. Jarchow, On certain locally convex topologies on Banach spaces, in: K. D. Bierstedt and B. Fuchssteiner (eds.), Functional Analysis: Surveys and Recent Results III, Math. Studies 90, North-Holland, Amsterdam 1984, 79-93. MR 85m:47050

[11] H. Jarchow, Weakly compact operators on $C(K)$ and $C^{*}$-algebras, in: H. Hogbe-Nlend (ed.), Functional Analysis and its Applications, World Sci., Singapore 1988, 263-299. MR 89m:46019

[12] H. Jarchow and U. Matter, On weakly compact operators on $C(K)$-spaces, in: N. Kalton and E. Saab (eds.), Banach Spaces (Proc., Missouri 1984), Lecture Notes in Math. 1166, Springer, Berlin 1985, 80-88. CMP 18:08

[13] J. Mujica, Complex Analysis in Banach Spaces, Math. Studies 120, North-Holland, Amsterdam 1986. MR 88d:46084

[14] A. Pietsch, Operator Ideals, North-Holland Math. Library 20, North-Holland, Amsterdam 1980. MR 81j:47001

Departamento de Matemáticas, Facultad de Ciencias, Universidad de Cantabria, 39071 SANTANDER, SPAIN

E-mail address: gonzalem@ccaix3.unican.es

Departamento de Matemáticas, ets de Ingenieros Industriales, Universidad PolitécNica de Madrid, C. José Gutiérrez Abascal 2, 28006 Madrid, Spain

E-mail address: jgutierrez@math.etsii.upm.es 